\title{
A Nudging Approach to Promote Healthier and More Sustainable Food Consumption and Lifestyles at the University of Milano-Bicocca
}

\author{
Matteo Colleoni $i^{1,}$, Massimiliano Rossetti ${ }^{2}$, Giacomo Magatti ${ }^{3}$, Paola Palestini ${ }^{4}$ Giovanna \\ lannantuoni ${ }^{5}$ \\ 1,2,3,4,5 University of Milano-Bicocca, Piazza dell'Ateneo Nuovo 1, 20126 Milan, Italy \\ * corresponding author: matteo.colleoni@unimib.it
}

Article Info

Received:

15 March 2021

Accepted:

25 May 2021

Published:

1 August 2021

DOI:

Presented in The $6^{\text {th }}$ International (Virtual) Workshop on UI GreenMetric World University Rankings (IWGM 2020)

\begin{abstract}
Since it was founded in 1998, the University of Milano-Bicocca has worked to make its structures environmentally, socially, and economically sustainable, not only to reduce the costs and environmental impact of its management processes, but also to promote sustainable behaviour on the part of its employees, lecturers and students. This report focuses on the measures implemented by the university in order to make food consumption and lifestyles healthier and more sustainable. Inspired by nudge theory (according to which in order to achieve a change in behaviour it is necessary to act on indirect encouragement and enablement rather than on direct instruction, enforcement and punishment), these measures aimed to promote healthier and more appropriate styles of food consumption, in particular at university canteens. The interventions were oriented both towards offering healthier products and towards modifying the environment in order to encourage more balanced food choices. The programme of interventions was divided into three phases: firstly an investigation of eating behaviours and lifestyles; secondly planning the interventions; and finally evaluating the project and disseminating good practices.
\end{abstract}

\section{Keyword:}

Sustainability, Food Policy, Choice Architecture, Behaviour.

\section{Introduction}

BASE (Bicocca Ambiente Società Economia - Bicocca Environment Society Economy) is an internal university structure that was launched to promote interaction between research and training, and which stimulates action on sustainability both within the university and outside it. At the university this interaction is exemplified by studies and research into sustainability, sustainability training for students and staff, and coordinating the various university sectors for the implementation of sustainability measures. Outside the university, 
meanwhile, BASE supports interest in sustainability by participating in working groups at a local level (the Bicocca District), nationally (the University Network for Sustainable Development), and internationally (the International Sustainable Campus Network - ISCN). BASE promotes a holistic approach to sustainability that includes a commitment to education on energy, waste, mobility, climate change, water, food, and sustainability itself. This report focuses on the measures implemented by the university in order to make food consumption and lifestyles healthier and more sustainable. Inspired by nudge theory (according to which in order to achieve a change in behaviour it is necessary to act on indirect encouragement and enablement rather than on direct instruction, enforcement and punishment), these measures aimed to promote healthier and more appropriate styles of food consumption, in particular at university canteens. The interventions were oriented both towards offering healthier products and towards modifying the environment to encourage more balanced food choices. The programme of interventions was divided into three phases: firstly an investigation of eating behaviours and lifestyles; secondly planning the interventions; and finally evaluating the project and disseminating good practices.

\section{Theoretical framework}

Eating behaviour and culture are closely linked; indeed, eating is one of the main aspects that define a culture. There are many different cultures and traditions spread across the world, and with them an incredible number of eating patterns, yet some problems, such as non-communicable diseases (NCD), can be found in many countries and even continents. Obesity is one such NCD, and represents a public health problem in many different countries. Although Italy's obesity problem can be considered mild in comparison to many of its neighbours (the country boasts one of the lowest adult obesity rates in Europe, with approximately $10 \%$ of the population obese in 2014), childhood obesity rates are notoriously considered to be among the highest (36\% for boys and $34 \%$ for girls). In addition, WHO projections predict that by 2030 rising prevalence could see disease rates nearly double for certain populations (Eurostat, 2019). A major problem influencing this is changes in lifestyle. People are spending less time at home, and eating has lost its importance, meaning out-of-home eating has been gaining in importance in recent decades. Catering outlets are an important setting for out-of-home eating as they have a vast reach across the population, and they therefore play a key role in tackling obesity. Altering environmental cues through choice architecture interventions has the potential to support the consumption of healthier food.

The main question with which we started to develop this project was How we can make a difference, or at least how can we try to make one? To begin with, a few simple definitions will help to better understand the decision-making process. There are essentially two possible approaches: the first is Neoclassical Economics, which emerged in the $20^{\text {th }}$ century to compete with the earlier theories of Classical Economics and dictates how we should decide. And then there is Behavioural Economics, which tell us instead how we decide. The last few decades have witnessed the growing development of interventions in behaviour change at a global level. This diffusion has been made possible by synergy among various disciplines related to the behavioural sciences, in particular psychology, sociology and economics.

At the core of the Behavioural Economics theory is the idea that rationality is limited in the decision-making process (Bounded Rationality). Rationality is limited by the tractability of the decision problem, the cognitive limitations and the time available. 
Decision-makers, according to this theory, act as satisficers, seeking a satisfactory solution rather than an optimal one. The winner of the 1978 Nobel Prize for economics, Herbert A. Simon, proposed bounded rationality as an alternative basis for the mathematical modelling of decision-making. It complements "rationality as optimisation", which views decisionmaking as a fully rational process of finding an optimal choice given the information available (Gigerenzer and Selten, 2002). Simon used the analogy of a pair of scissors, where one blade represents human cognitive limitations and the other the "structure of the environment", illustrating how minds compensate for limited resources by exploiting known structural regularity in the environment. One use of this idea can be found in Sustein and Thaler's Nudge (2008). As Thaler, the winner of the 2017 Nobel Prize for economics, stated: "A nudge [...] is every aspect of the choice architecture that modifies the behaviour of people in a predictable way, without preventing alternative choices or significantly changing their economic incentives". In their perspective, a nudge should fulfil the following conditions: $i$ ) it must be ethically and morally acceptable; ii) freedom of choice must still be provided and iii) it must be easy to avoid.

Although some critics of nudge have claimed that modifying choice architectures will lead to people becoming worse decision-makers, the university supported the application of nudge theory within the university canteen with the aim of increasing the likelihood of people opting for the healthiest food choice, instead of a less healthy option. The aim of this pilot trial was to measure the effect of nudge strategies in the university canteen so as to better understand the links between nudging and food choices.

\section{Developing a university-wide food policy : the nudging programme at the University of Milano-Bicocca}

Growing attention has been paid in recent years to studying living and working conditions in professional environments, and to improving the quality of life of the people who spend a considerable proportion of their day there. It is thought that the way spaces are organised and, more generally, the characteristics of the environments in which the main social activities take place can facilitate positive behaviours that lead to improved personal wellbeing. This is particularly true for behavioural studies relating to nudge theory and choice architecture, as we saw in the previous paragraph, which aim to explore the interactions between contextual aspects and changes in behaviour. Of all the consumption behaviours studied through these theories, food consumption has received particular attention.

This project, in collaboration with IESCUM (European Institute for the Study of Human Behaviour) and Sodexo, aimed to promote healthy and sustainable lifestyles for the staff and students of the University of Milano-Bicocca. The project was divided into three phases. The first involved analysing the eating habits of students and staff (both teaching and technical/administrative staff) in relation to the features of the environments where the food is consumed. The second, in light of the survey results, saw work carried out to restructure the spaces and the environments in which food is provided. Finally, the third involved evaluating and publicising the results of the intervention.

\subsection{Eating at Bicocca. Types of user, places and opinions.}

The research, conducted by BASE, firstly sought to analyse the eating habits of students and staff (both teaching and technical/administrative staff) in relation to the characteristics of the environments where the food is consumed. It also aimed to provide 
empirical evidence that would form the basis for interventions to reorganise the spaces and environments in which food is offered, in order to promote food consumption behaviours that are healthier for the individual and more environmentally sustainable.

The survey was conducted using an online, self-administered questionnaire, split into three sections, which in turn were divided up as follows:

- canteen users and the types of people attending it;

- opinions on the canteen (food - spaces - service - menu - value - satisfaction);

- reasons for not using the canteen (food - spaces - motivation).

A sample of 2,782 people responded to the survey, administered between December 2018 and January 2019, of whom 68\% were women and 32\% were men. The university population can be divided into those who use the university's catering facilities (52\%) and those who do not (48\%). The BMI (Body Mass Index) distribution of the population does not highlight any major issues, as the population under investigation contains a large number of young people. Overall, $11.7 \%$ of people are underweight, $76.6 \%$ have a normal weight, and $10.4 \%$ and $1.3 \%$ are overweight and obese respectively. Although the data are not a cause for concern, they show a general trend of women towards being underweight and men towards being overweight (see table 1 ).

Table.1. BMI of the population of the University of Milano-Bicocca by gender and role.

\begin{tabular}{cccccc}
\hline & & Students & Lecturers & Staff & Total \\
\hline \multirow{4}{*}{ Female } & Underweight & $16.2 \%$ & $3.5 \%$ & $3.8 \%$ & $15.6 \%$ \\
& Normal & & & & \\
& Weight & $74.2 \%$ & $80.6 \%$ & $81.0 \%$ & $74.7 \%$ \\
& Overweight & $8.3 \%$ & $13.5 \%$ & $11.4 \%$ & $8.4 \%$ \\
& Obese & $1.3 \%$ & $2.4 \%$ & $3.8 \%$ & $1.3 \%$ \\
\hline \multirow{5}{*}{ Male } & $100.0 \%$ & $100.0 \%$ & $100.0 \%$ & $100.0 \%$ \\
\hline & Underweight & $3.6 \%$ & & & $3.4 \%$ \\
& Normal & & & & \\
& Weight & $82.5 \%$ & $71.6 \%$ & $49.8 \%$ & $80.9 \%$ \\
& Overweight & $12.7 \%$ & $26.7 \%$ & $43.4 \%$ & $14.4 \%$ \\
& Obese & $1.2 \%$ & $1.7 \%$ & $6.8 \%$ & $1.3 \%$ \\
\hline
\end{tabular}

Focusing on the users of these services, factor analysis was used to construct four satisfaction indices for the canteens at Bicocca, while cluster analysis highlighted four types of user of the canteen service. The majority of respondents eat a packed lunch on university premises (53\%) or buy lunch from the canteen (52\%). A significant proportion return home to eat lunch (22\%) or use cafes/restaurants outside the university $(20 \%)$. The latter are more popular than the university cafes, which are used by just $10 \%$ of respondents.

\subsubsection{Canteen customers and the types of people attending it}

The majority of students eat a packed lunch on university premises (55\%), especially women ( $62 \%$ versus $37 \%$ of men). The canteen is chosen by $52 \%$ of students, $60 \%$ of men and $48 \%$ of women.

While students receiving scholarships prefer to get food from the canteen (84\%), those who don't prefer a packed lunch on university premises (63\%). Seven out of every 100 
students skip the meal entirely, while one in four return home to eat lunch.

The majority of lecturers eat lunch in the canteen (51\%), followed by cafes/bars outside the university (41\%) and university cafes (22\%). A smaller proportion, around $17 \%$, eat a packed lunch on university premises, while $11 \%$ skip the meal altogether. The canteen is used slightly more by men, while the cafes and restaurants outside the university are favoured by women. Like the lecturers, the majority of technical and administrative staff $(64 \%)$ eat lunch in the canteen, followed by cafes/bars outside the university (49\%). In third place is a packed lunch eaten in the university's various facilities (22\%). Only four respondents in 100 skip the meal, with no distinction between the genders.

Top of the list of the main reasons given by respondents for why they use the canteen is its vicinity to their place of study/work (58.5\%) followed by the availability of incentives such as vouchers/scholarships (41.2\%) and the affordable price (36.9\%). A similar proportion of respondents visit the canteen because they believe the food is satisfactory (31.6\%) and the service is fast (31.4\%). Students who do not receive scholarships present values that are significantly higher than average for the affordable price and speed of service. Students in receipt of scholarships, meanwhile, give the fact that they receive a scholarship as their main motivation. Lecturers appreciate the short distance from their workplace and the speed of service. Finally, technical and administrative staff present above-average values for the canteen's proximity to their workplace and for possessing vouchers.

Based on the reasons people gave for visiting the canteen, four types of attendee where identified:

- Frugal: those who attend the canteen because they have vouchers or a scholarship. This category predominantly comprises students with scholarships and staff.

- Pragmatic: those who pay most attention to the value provided by the service. This group states that they visit the canteen because the food is satisfactory (74\%) and inexpensive (97\%), and is mostly made up of students without scholarships.

- Hurried: those who visit the canteen because it is quick (100\%) and nearby $(77 \%)$. This type of user comprises lecturers as well as students without scholarships.

- Creatures of habit: those who go to the canteen because, as well as being nearby $(74 \%)$, it is used by colleagues and friends $(53 \%)$, or simply out of habit $(28 \%)$. This last type does not present any particular differences between the roles.

\subsubsection{Opinions of the canteen}

Those who make use of the university canteen were invited to indicate their level of satisfaction regarding the food, premises, service and menu. The service presents the highest satisfaction rating, with $81 \%$ declaring themselves satisfied. This is followed by the premises $(69 \%)$ and food $(67 \%)$, which are broadly similar in terms of overall satisfaction. The menu has the highest levels of dissatisfaction, with $57 \%$ of users critical of it. Specifically, $60 \%$ say they are not satisfied with the food options for those with allergies and intolerances and $55 \%$ are dissatisfied with the vegetarian or ethnic food options. Another negative point highlighted is the way the food is arranged along the service line, which has a $50 \%$ dissatisfaction level. Opinions on the information available on the ingredients used were slightly better (positive in $53 \%$ of cases).

In general, despite the detailed information highlighting certain negative aspects, users rate the university's catering service positively. $74 \%$ say they are satisfied and $72 \%$ show significant appreciation of the value offered. 


\subsubsection{Reasons for not using the canteen}

People's reasons for not using the canteen vary depending on their role within the university. Students who do not receive scholarships present above-average values for the options "I prefer the food I bring from home" (42\%) and "excessive cost" (32.4\%). Students who receive scholarships, meanwhile, cite reasons relating to long waiting times (34.9\%) and lacking time (37.3\%). Lecturers express negative opinions on the quality of the food (78.8\%), poor value (60.6\%) and overcrowding (54.5\%). Finally, technical and administrative staff present above-average values for the options "low food quality" (70\%), "poor value" $(50 \%)$ and "long waiting times" (35\%). Of the less frequently cited reasons, lecturers present above-average values for the options regarding smell, noise, service and the antisocial nature of the canteen.

Gender does not have a significant impact on the reasons given for choosing not to use the canteen. The main exceptions to this refer to the decision to bring a packed lunch, preferred by women ( $45.1 \%$ compared to $27.4 \%$ of men), the waiting time ( $30 \%$ of women versus $19 \%$ of men), a lack of time (chosen by $28.7 \%$ of women compared to $19.3 \%$ of men) and finally the excessive cost (stated more by men at $36.6 \%$, compared to $28.3 \%$ of women).

\subsection{Work to design a healthy and sustainable canteen.}

Although the survey revealed that, on average, people view the canteen positively, certain negative aspects highlighted by the results demanded further investigation. In particular, the layout of the service line and the presentation of the foods, the elements that saw the lowest level of satisfaction among respondents, were placed under observation. This phase involved the people Thaler and Sustein (2008) call "choice architects", those who design interventions with the aim of altering behavioural choices.

Specifically, with the support of IESCUM, the environment in the canteen in one of the university buildings was reorganised, with measures introduced designed to change people's behaviour. These focused on altering the service line in the canteen to encourage people to choose healthy and nutritionally balanced foods.

The intervention was carried out in three phases, between April and June 2019:

- Week 1 - Baseline (8-12 April). A video camera was placed at the till to record the contents of trays and to find out users' choices in terms of the calories consumed per meal and how varied and healthy their diets are.

- Week 2 - Intervention (6-10 May). The "choice architects" then intervened, focusing on the following actions:

a) Simplified information: simplifying the information, with the aim of reducing the time needed to work out the content of foods and to make the relevant choices. Adding a "so good" sticker and coloured signs for fruit, vegetables and wholemeal bread (see figure 1) made choosing foods simpler and made the associated information easier to understand.

b) Increased prominence: since fruit and vegetables were difficult to see in the original layout (see figure 1), certain changes were made in order to make them more visible and accessible. The containers holding them were raised by approximately $30 \mathrm{~cm}$ compared to their previous position, and "green footprints" were placed on the floor to guide users towards the fruit and vegetable sections (which were also marked by the "so good" sticker).

c) Framing effect: this involves changing the ways in which the information or products are presented, without changing the options available. Figure 1 shows 
how the layout of the fruit salad was altered - it was moved to a position where it was more likely to be seen. Other changes included separating the white bread from the wholemeal bread and placing the latter in front of the former. In addition, the day's menu was shown at the entrance to the canteen and repeated along the service line, with the healthiest foods marked by the same quality sticker.

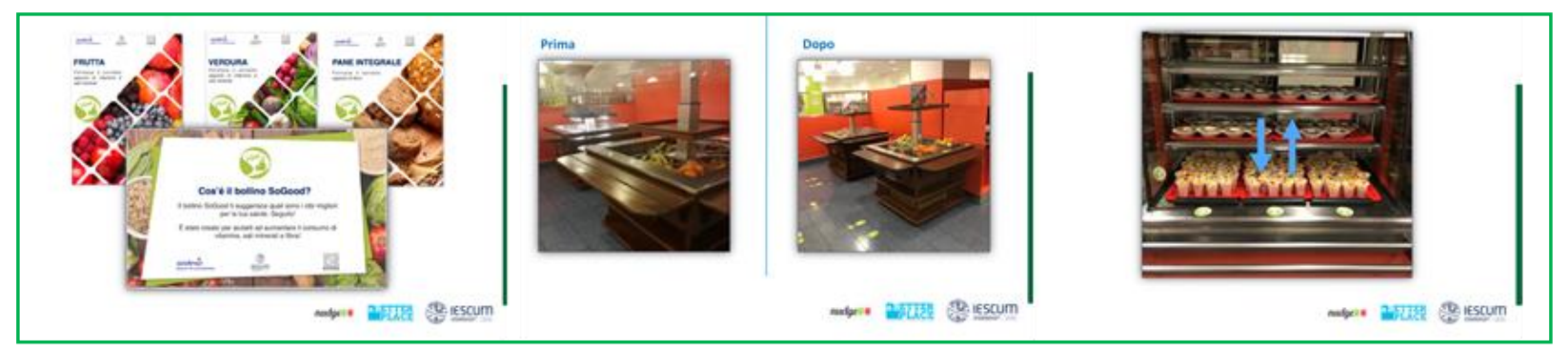

Figure.1. The intervention. Examples (from left to right) of simplified information, increased prominence and the framing effect.

- Week 3 - Follow-up (27-31 May). During the third week, the behavioural changes associated with the interventions made (the so-called "nudge") were observed and the length of time they would last was estimated.

\subsection{Evaluation and dissemination}

The project enacted in the university canteen highlighted the importance of the layout and presentation of food in people's food choices. More generally, the positive results obtained gave empirical value to theories on libertarian paternalism (Thaler and Sustein, 2008).

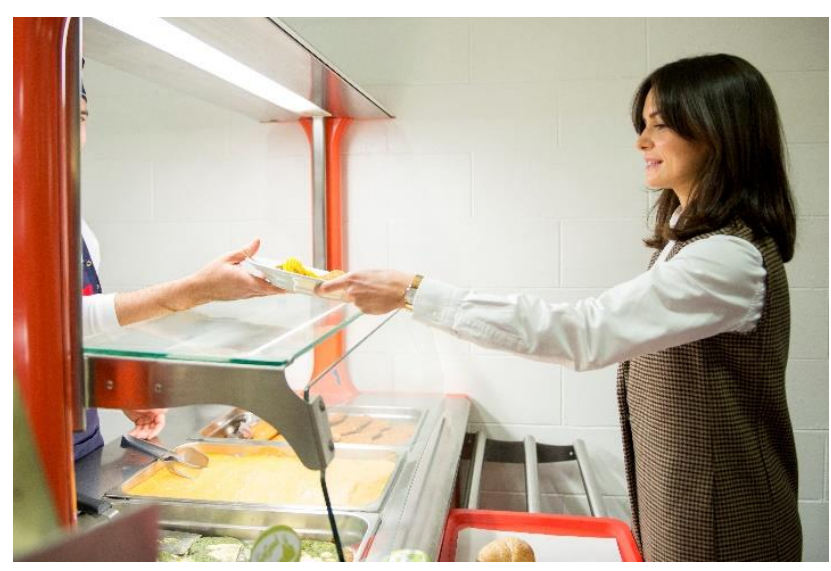

Figure 2. A user of the Building U6 canteen

Leaving behind the negative connotations of these two associated terms, the university therefore shows itself to be a favourable environment for a gradual, steered change in behaviour. In the first two phases of the project, 1,647 and 1,570 trays respectively and various target foods such as wholemeal bread, fruit, vegetables and items from the hot range were compared, and an increase of $28 \%$ was recorded between the baseline phase ( 0.89 foods per tray) and the intervention stage (1.19). Wholemeal bread, initially found on $6 \%$ trays, once separated from the rest of the bread and placed further forwards, recorded the most significant increase, up $200 \%$. Creating guided pathways 
towards the most attractive sections saw a $50 \%$ increase in consumption of raw vegetables (compared to their initial appearance in just $6 \%$ of trays). Consumption of fruit and foods from the hot range also increased, accounting for $18 \%$ and $69 \%$ of trays respectively (an increase of approximately $13 \%$ ).

In conclusion, the positive results achieved supported extending the projects to other canteens and cafes in the university during the following academic year. The project's main results were communicated at various national and international conferences (including the ISCN Conference 2019 in São Paolo and FENS 2019, the 13th European Nutrition Conference, in Dublin).

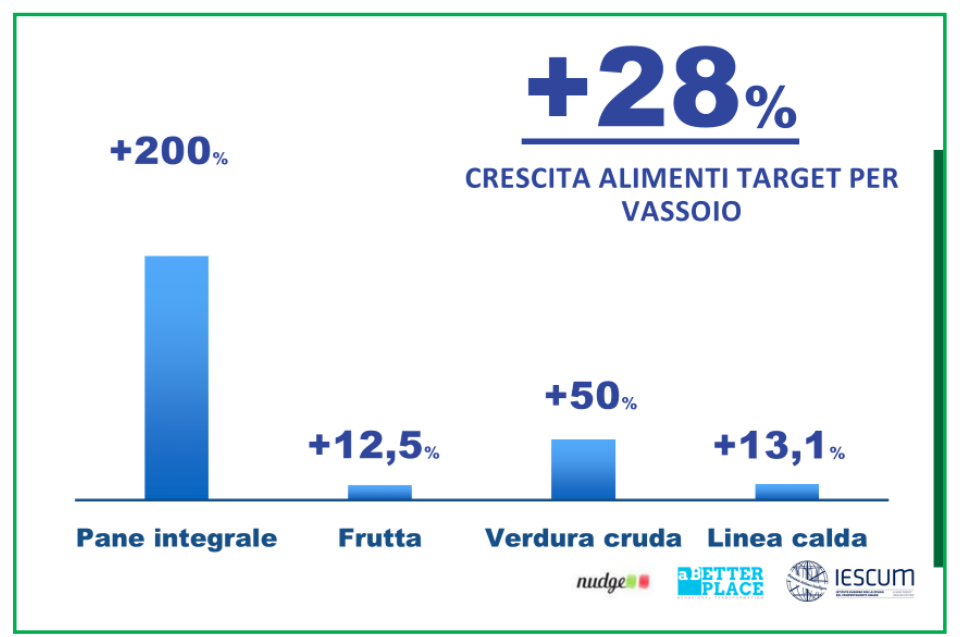

Figure 3. Measuring the intervention

\section{Conclusions}

This article has highlighted the main results of a project by the University of MilanoBicocca that sought to use the nudging approach developed in the field of "choice architecture" to promote certain eating habits among students and staff and therefore increase their level of wellbeing. The positive results obtained through the interventions made, in terms of increased consumption of healthy foods and more varied diets, support the value of the nudging policies tested. The contextual change of the spatial layout of the range of foods, combined with the use of more effective communication and information tools, encouraged people to move towards healthier and more varied choices. The increased variation in choices and therefore more varied menus also reduced the amount of unused food, connecting the project with another one related to food waste, conducted in collaboration with the Department of Informatics, System and Communication, which aims to analyse the quantity and type of food left on trays, as well as to eliminate single-use plastic from the university canteens. Another measure taken in this respect was handing out over 12,000 drink bottles and installing tap water dispensers, with the aim of gradually eliminating the use of plastic bottles.

Finally, it should be noted that a University Catering Committee was set up, entrusted by the Rector to govern all food policy interventions and to draw up an action plan in the food sector, as part of the University of Milano-Bicocca's General Sustainability Plan.

\section{References}

1. Boffi M., Colleoni M., Scolari G., Rossetti M. (2019), Mangiare in Bicocca i luoghi della 
ristorazione. Technical Report coordinated by Paola Palestini, chair of the University Catering Committee.

2. Eurostat, 2019: https://appsso.eurostat.ec.europa.eu/nui/submitViewTableAction.do. Accessed 10.08.2019

3. Gigerenzer, Gerd; Selten, Reinhard (2002). Bounded Rationality: The Adaptive Toolbox. MIT Press. Hollands G et al., 2013: Altering micro-environments to change population health behavior: towards an evidence base for choice architecture interventions. BMC Public Health 13:1218

4. Maxwell, S.; Slater, R. (2003). "Food policy old and new". Development Policy Review. 21 (5-6): 531-553

5. Simon, Herbert A. (1947) Administrative Behavior: a Study of Decision-Making Processes in Administrative Organization. Macmillan

6. Simon, Herbert A. (1956). Rational Choice and the Structure of the Environment. Psychological Review. 63 (2): 129-138.

7. Thaler, R.H., \& Sunstein, C.R. (2008). Nudge: Improving Decisions about Health, Wealth, Happiness. New Haven, CT: Yale University Press. 